\title{
Induction of Dickkopf-1, a Negative Modulator of the Wnt Pathway, Is Associated with Neuronal Degeneration in Alzheimer's Brain
}

\author{
Andrea Caricasole, ${ }^{1 *}$ Agata Copani, ${ }^{2,6 *}$ Filippo Caraci, ${ }^{2}$ Eleonora Aronica, ${ }^{3}$ Annemieke J. Rozemuller, ${ }^{3}$ \\ Alessandra Caruso, ${ }^{4}$ Marianna Storto, ${ }^{5}$ Giovanni Gaviraghi, ${ }^{1}$ Georg C. Terstappen, ${ }^{1}$ and Ferdinando Nicoletti ${ }^{4,5}$ \\ ${ }^{1}$ Siena Biotech, 53100 Siena, Italy, ${ }^{2}$ Department of Pharmaceutical Sciences, University of Catania, 95125 Catania, Italy, ${ }^{3}$ Department of Neuropathology, \\ Academic Medical Center, Univeristy of Amsterdam Meibergdreef, Amsterdam 1105 AZ, The Netherlands, ${ }^{4}$ Department of Human Physiology and \\ Pharmacology, University of Rome La Sapienza, 00185 Rome, Italy, ${ }^{5}$ Istituto Neurologico Mediterraneo, Neuromed, 86077 Pozzilli, Isernia, Italy, and \\ ${ }^{6}$ Istituto di Bioimmagini e Biostrutture, CNR-95125 Catania, Italy
}

We used primary cultures of cortical neurons to examine the relationship between $\beta$-amyloid toxicity and hyperphosphorylation of the tau protein, the biochemical substrate for neurofibrillary tangles of Alzheimer's brain. Exposure of the cultures to $\beta$-amyloid peptide $(\beta \mathrm{AP})$ induced the expression of the secreted glycoprotein Dickkopf-1 (DKK1). DKK1 negatively modulates the canonical Wnt signaling pathway, thus activating the tau-phosphorylating enzyme glycogen synthase kinase- $3 \beta$. DKK1 was induced at late times after $\beta A P$ exposure, and its expression was dependent on the tumor suppressing protein p53. The antisense induced knock-down of DKK1 attenuated neuronal apoptosis but nearly abolished the increase in tau phosphorylation in $\beta$ AP-treated neurons. DKK1 was also expressed by degenerating neurons in the brain from Alzheimer's patients, where it colocalized with neurofibrillary tangles and distrophic neurites. We conclude that induction of DKK1 contributes to the pathological cascade triggered by $\beta$-amyloid and is critically involved in the process of tau phosphorylation.

Key words: Alzheimer's disease; $\beta$-amyloid; Wnt pathway; Dickkopf-1; tau phosphorylation; apoptosis

\section{Introduction}

Evidence from human genetics and transgenic mice suggests that an overproduction of $\beta$-amyloid peptide $(\beta \mathrm{AP})$ is a primary event in the pathophysiology of Alzheimer's disease (AD) (Hardy and Selkoe, 2002). $\beta$ AP applied to neuronal cultures induces apoptosis (Loo et al., 1993), a phenotype of death that is also observed in the AD brain (Cotman and Anderson, 1995; Smale et al., 1995). How this can be reconciled with the formation of neurofibrillary tangles (NFTs) is uncertain because, at least in vitro, neurons exposed to $\beta \mathrm{AP}$ die too rapidly to allow the formation of NFTs. NFTs might arise in neurons that in vivo escape a fast execution of apoptotic death (Caricasole et al., 2003).

DNA damage associated with p53 expression may be a point of convergence of multiple intracellular pathways related to $\beta \mathrm{AP}$ toxicity (Zhang et al., 2002). p53 induces a number of genes that promote either DNA repair or apoptotic death (Lakin and Jackson, 1999; Shen and White, 2001). We wondered whether a p53

\footnotetext{
Received Feb. 2, 2004; revised May 19, 2004; accepted May 20, 2004.

This work was supported by a grant from the Alzheimer's Association. We thank Elisa Trovato Salinaro for technical assistance and Dr. Peter Davies for kindly providing the PHF-1 antibody.

${ }^{*}$ A. C. and A. C. contributed equally to this work.

Correspondence should be addressed to Dr. Ferdinando Nicoletti, Department of Human Physiology and Pharmacology, University of Rome La Sapienza, Piazzale Aldo Moro 5, 00185 Rome, Italy. E-mail: ferdinandonicoletti@hotmail.com.

D0I:10.1523/JNEUROSCI.1381-04.2004

Copyright $\odot 2004$ Society for Neuroscience $\quad 0270-6474 / 04 / 246021-07 \$ 15.00 / 0$
}

transcription program is involved in the formation of NFTs in $\beta \mathrm{AP}$-treated neurons. Formation of NFTs results from a hyperphosphorylation of the tau protein, which is potentially driven by glycogen-synthase kinase-3 $\beta$ (GSK3 $\beta$ ) (Takashima et al., 1998; Otth et al., 2002), an enzyme that is under the control of the canonical Wnt signaling pathway (Grimes and Jope, 2001). This pathway is activated by different Wnt secreted glycoproteins that interact with the Frizzled and LRP5/6 (LDL receptor-related protein type 5 and 6) membrane coreceptors (Dale, 1998). Activation of the Wnt pathway leads to inhibition of GSK3 $\beta$ by dissociating the enzyme from a multiprotein complex that involves axin, adenomatous polyposis coli, and $\beta$-catenin (Willert and Nusse, 1998 ) and via phosphorylation of GSK3 $\beta$ on $\operatorname{Ser}_{9}$ (Fukumoto et al., 2001). This results in the stabilization of the underphosphorylated form of $\beta$-catenin, which is no longer targeted for degradation by the proteasome and is then made available for its transcriptional and cell adhesion functions (Hinck et al., 1994; Willert and Nusse, 1998). Recent evidence suggests that a loss of Wnt function is implicated in the pathophysiology of neuronal degeneration in AD (De Ferrari and Inestrosa, 2000; Garrido et al., 2002; Inestrosa et al., 2002; De Ferrari et al., 2003). The canonical Wnt pathway is negatively modulated by the extracellular protein Dickkopf-1 (DKK1), which binds to LRPs preventing their interaction with Wnts (Zorn, 2001). DKK1 is induced by p53 (Wang et al., 2000) and might therefore be a component of the sequence of events leading to neuronal toxicity in response to 
$\beta A P$. Induction of DKK1 would prevent the inhibition of GSK3 $\beta$ by Wnt, thus facilitating phosphorylation of tau protein and formation of NFTs in neurons that survive a rapid execution of apoptotic death. We now report that DKK1 is expressed in cultured cortical neurons exposed to $\beta \mathrm{AP}$ as well as in neurons from autoptic brain samples of AD patients. In addition, we show that $\beta \mathrm{AP}$-induced DKK1 expression is under the control of $\mathrm{p} 53$ and is causally related to hyperphosphorylation of tau in neurons challenged with $\beta$ AP.

\section{Materials and Methods}

Culture preparation, treatments, and assessment of neuronal death. All experiments were performed in compliance with the European Union use of laboratory animals, the guidelines of the Italian Decreto Legislativo 27/1/92, number 116, article 7, and according to the institutional approved protocol number 9213. Cultures of pure cortical neurons were obtained from embryonic day (E) 15 rat embryos according to a well established method that allows the growth of a $>99 \%$ pure neuronal population (Copani et al., 1999). $\beta \mathrm{AP}(25-35)$ was purchased from Bachem Feinchemikalien AG (Bubendorf, Switzerland). Different lots of the peptide were used. $\beta$ AP (25-35) was solubilized in sterile, doubly distilled water at an initial concentration of $2.5 \mathrm{~mm}$ and stored frozen at $-20^{\circ} \mathrm{C}$. It was used to a final concentration of $25 \mu \mathrm{M}$ in the presence of the glutamate receptor antagonists MK-801 [(+)-5-methyl-10,11dihydro-5H-dibenzo [a,d] cyclohepten-5,10-imine maleate] (10 $\mu \mathrm{M})$ and DNQX $(30 \mu \mathrm{M})$ to avoid the potentiation of endogenous glutamate toxicity (Copani et al., 1999). In some experiments, cultures were treated with the following "end-capped" phosphorothioate antisense (As) oligonucleotides: p53-As, 5'-gacctcaggtggctcatacgg-3'; p53-Sense (Sn), 5' ccgtatgagccacctgaggtc-3' (Chen et al., 1999); DKK-As, 5'-cgtcggagggag gcgagc- $3^{\prime}$; DKK-Sn, 5' -gctcgcctccctccgacg-3'. Oligonucleotides (3 $\mu \mathrm{M}$; MWG-Biotech, Firenze, Italy) were applied to cultures $16 \mathrm{hr}$ before the addition of $\beta$ AP. Assessment of neuronal death was performed by combining MTT assay and cytofluorimetric analysis of hypoploid DNA (Copani et al., 1999; Copani et al., 2002).

Reverse transcriptase-PCR and real-time PCR analysis. Total RNA was extracted from the cultures as described previously (Godemann et al., 1999). Total RNA was subjected to DnaseI treatment (Roche, Hertforshire, UK) and $2 \mu \mathrm{g}$ of total RNA/sample were used for cDNA synthesis using Superscript II (Invitrogen, San Diego, CA) and an oligodT primer. Each reverse transcriptase (RT) product was diluted to $100 \mu$ l with sterile, distilled water, and $1 \mu \mathrm{l}$ of cDNA was used in the subsequent PCR amplification. $\beta$-actin cDNA amplification was performed by using a pair of primers (Roelen et al., 1994), which span an intron and yield products of different sizes depending on whether cDNA or genomic DNA is used as a template ( $400 \mathrm{bp}$ for a cDNA-derived product, and 600 bp for a genomic DNA-derived amplification). The following pairs of primers were used:

FZD1-FOR AAGTATGGCTGAGGAGGCGGT

FZD1-REV GGTCTGATTGTACGCGATGTC

FZD2-FOR CCATCATCTTCCTGTCCGGTT

FZD2-REV GGAAGTACTGCGAATTGGCCT

FZD3-FOR TATGGCTGGCAGTGTATGGTG

FZD3-REV TAATGCCGGCTAAGAGGAGAG

FZD4-FOR TAGTGGATGCCGATGAGCTGA

FZD4-REV TCCACATGCCTGAAGTGATGC

FZD5-FOR GTGCACAGTCGTCTTCCTCTT

FZD5-REV GCCTCGTAGCGAGTTCAGGTT

FZD6-FOR TTAGTGACGCTTCTTGGCTGC

FZD6-REV GCCATGCTTCTTCTTGTGCCT

FZD7-FOR TTACTTCTTCGGCATGGCCAG

FZD7-REV TCTTGGTGCCATCGTGCTTCA

FZD8-FOR CACAGCTGAGGAATGCCGAAT

FZD8-REV GGCTGCGATGAACATAGTGGA

FZD9-FOR GTGTTCACCTTCCTGCTGGAG

FZD9-REV AGCCAGGAACCAGGTGAGAGT

FZD10-FOR TTCTTCTCCAGCGCCTTCACC

FZD10-REV AGCTGGCCATGCCGAAGTAGT
Wnt1-FOR TCCTCCACGAACCTGCTGACA Wnt1-REV GTCGCAGGTGCAGGATTCGAT Wnt2-FOR TGGTGGTACATGAGAGCGACA Wnt2-REV AATACAACGCCAGCTGAGGAG Wnt2B-FOR ACCTGAGGAGGCGATATGATG Wnt2B-REV ACAGCACAGCACCAGTGGAAT Wnt3-FOR CTGGTGTAGCCTTGCCAGTCA Wnt3-REV CTTCACCTCACAGCTGCCAGA Wnt3A-FOR CATCGCCAGTCACATGCACCT Wnt3A-REV CGTCTATGCCATGCGAGCTCA Wnt4-FOR GAAGGCCATCCTGACACACAT Wnt4-REV ACACCAGGTCCTCATCCGTAT Wnt5A-FOR AAGCAGGTCGCAGGACAGTAT Wnt5A-REV TCTGAGGTCTTGTTGCACAGG Wnt5B-FOR GGAACTGACCAACAGCCGCTT Wnt5B-REV GGTCCACGATCTCGGTGCATT Wnt6-FOR GCTGCGGAGATGATGTCGACT Wnt6-REV GAATCGGCTGCGTAGAGGAGA Wnt7B-FOR CAAGGAGAAGTACAACGCAGC Wnt7B-REV CACTTGACGAAGCAACACCAG Wnt8A-FOR CGCAGAGGCTGAGCTGATCTT Wnt8A-REV CACACTTGACCGTGCAACACC Wnt9A-FOR AGCACTACCAATGAAGCCACC Wnt9A-REV ACACTGCCTGCACTCCACATA Wnt10A-FOR TCACTCCGACCTGGTCTACTT Wnt10A-REV CTCAGTGATGCGGCATTCTTC Wnt10B-FOR AGAGTGCGTTCTCCTTCTCCA Wnt10B-REV CATGTCGTGATTACAGCCACC Wnt11-FOR AAGTGGTACACCGGCCTATGG Wnt11-REV TCACTTGCAGACGTAGCGCTC Wnt16-FOR CAGTACGGCATGTGGTTCACG Wnt16-REV CTCTCCTGCGCATCTTCCTCT LRP5-FOR GGCCAGTGGTCCCTTTCC LRP5-REV GGCTATGAAGTTGAGAGGCAC LRP6-FOR AGCGGCAGTGCATTGA

LRP6-REV ATCCGATTTATCCTGGCAGTT DKK1-FOR AATCTGCCTGGCTTGCCGAA DKK1-REV GTGGAGCCTGGAAGAATTGC

Real-time PCR was performed using an I-Cycler and the iQ SYBR Green Supermix (Bio-Rad, Hercules, CA) according to the instructions of the manufacturer. Primer sequences were designed to different exons of the rat DKK1 and $\beta$-actin genes. Primer sequences were as follows: DKK1-FOR 5'- GCTGCATGAGGCACGCTAT-3'; DKK1-REV $5^{\prime}$ AGGGCATGCATATTCCGTTT- ${ }^{\prime} ; \beta$-actin-FOR $5^{\prime}$ - CCCTGGCTCCTAGCACCAT- $3^{\prime} ; \beta$-actin-REV $5^{\prime}$-GAGCCACCAATCCACACAGA$3^{\prime}$. Reaction conditions were as follows: $95^{\circ} \mathrm{C} / 5^{\prime} ; 35 \times\left(95^{\circ} \mathrm{C} / 30^{\prime} ; 55^{\circ} \mathrm{C} /\right.$ $\left.30^{\prime}\right)$. Results were analyzed with the I-Cycler Optical System software version 3.0a and represented as means \pm SEM.

$T$-cell factor/lymphoid enhancer factor-based luciferase reporter studies. Transfections and reporter assays were performed essentially as described previously (Caricasole et al., 2002). Transient transfections of primary cortical neurons were performed in triplicate using Lipofectamine 2000 (Invitrogen). Neurons $\left(\sim 3 \times 10^{5}\right.$ per well in 24 -well plates) were transfected at $12 \mathrm{~d}$ in vitro. A total of $1.02 \mu \mathrm{g}$ of DNA was transfected into each well, including luciferase reporter plasmid (200 ng), expression construct (250-800 ng of each expression construct, for up to two different plasmids), Renilla luciferase cytomegalovirus-driven internal reporter (20 ng; Promega, Madison, WI), and carrier plasmid DNA (pBluescript; Promega; to $1.02 \mu \mathrm{g}$ ) as appropriate. The luciferase reporter plasmid was the $\mathrm{p} 4 \mathrm{TCF}$, comprising four copies of a T-cell factor (TCF) responsive element upstream of a TATA element-luciferase coding sequence transcriptional unit (Bettini et al., 2002). After replacement of transfection medium with culture medium, $\beta$ AP was added to a final concentration of $25 \mu \mathrm{M}$ to wells, as appropriate. Luciferase activity was measured using the Promega Dual Luciferase Assay Reagent and read using a Berthold (Bad Wildbad, Germany) LUMAT LB3907 tube luminometer. Readings were from triplicate transfections and were automatically normalized relative to the internal standard (Renilla luciferase).

Western blot analysis. Western blot analysis was performed on total cell 
a

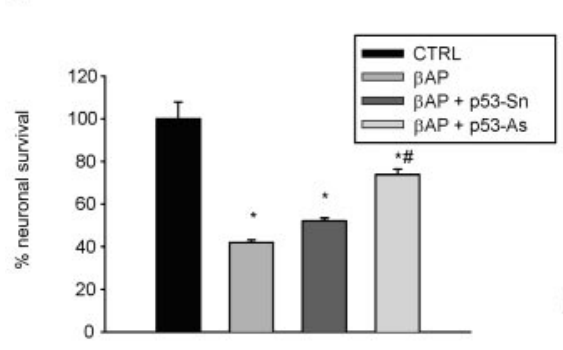

C
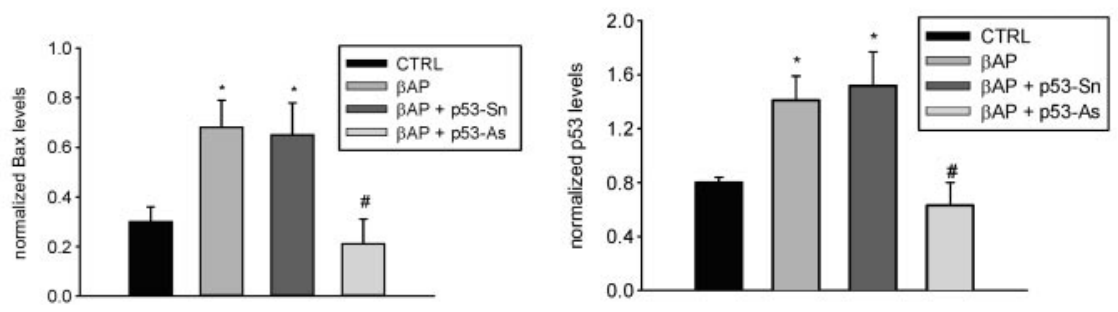

Figure 1. Protective effects of $p 53$ knock-down in pure neuronal cultures exposed to $\beta A P . a, p 53$ antisense oligonucleotides (p53-As) increase neuronal survival in cultures exposed to $25 \mu \mathrm{m} \beta A \mathrm{P}(25-35)$ for $24 \mathrm{hr}$. Values are means \pm SEM of four determinations. ${ }^{*} p<0.05$ versus controls (CTRL); ${ }^{*} p<0.05$ versus $\beta$ AP alone (one-way ANOVA followed by Fisher's LSD test). $b$, Representative immunoblots of Bax (top) and p53 (bottom) in protein extracts from cortical neurons that have been treated with $25 \mu \mathrm{m} \beta \mathrm{AP}(25-35)$ for $24 \mathrm{hr}$ in the absence or presence of $\mathrm{p} 53 \mathrm{As}$ or senses (Sn). c, d, Densitometer scan analysis from three determinations. Values represent the means \pm SEM of $\operatorname{Bax}(c)$ or p53 (d) normalized against $\beta$-actin in three independent experiments. ${ }^{*} p<0.05$ versus controls, or ${ }^{\#} \beta A P$ and ${ }^{\#} \beta A P$ plus $p 53-S n$ (one-way ANOVA followed by Fisher's LSD test).

extracts (Copani et al., 2002). The primary antibodies (Abs) were: antigoat DKK1 (1:100; Santa Cruz Biotechnology, Santa Cruz, CA), antimouse paired helical filament (PHF)-1 (1:100; kindly provided by Dr. Peter Davies, Albert Einstein College of Medicine, Yeshiva University, Bronx, NY), anti-mouse p53 (5 $\mu \mathrm{g} / \mathrm{ml}$; Oncogene Sciences, Uniondale, NY), anti-rabbit Bax (1:100; Santa Cruz Biotechnology), and anti-rabbit GSK3 $\beta$, phospho (ser9) (1:1000; Cell Signaling Technology, Beverly, $\mathrm{MA})$.

Immunohistochemistry. Human brain specimens were obtained at autopsy from the Department of Pathology of the Academic Medical Center (University of Amsterdam), the Vrije Universiteit Medical Center, and the Netherlands Brain Bank (R. Ravid, coordinator). Permission was obtained for performing autopsies, for the use of tissue, and for access to medical records for research purposes. Clinical diagnosis was neuropathologically confirmed on formalin-fixed, paraffin-embedded tissue from different sites. For this study, we used material from six AD patients and six age-related nondemented controls (see Fig. 6 legend). The average postmortem interval was $8.3 \pm 0.9 \mathrm{hr}$ for normal controls and $4.2 \pm$ $0.7 \mathrm{hr}$ for AD cases. Staging of AD was evaluated according to Braak and Braak (1995), and adjacent sections were immunostained for $\mathrm{A} \beta$, tau protein, and Congo red as described previously (Arends et al., 2000). Formalin-fixed $(4 \% ; 24 \mathrm{hr})$ paraffin-embedded tissue from the temporal and frontal cortex was used. Sections ( $5 \mu \mathrm{m}$ thick) were mounted on poly-L-lysine-coated tissue slides and deparaffinized. Subsequently, sections were immersed in $0.3 \% \mathrm{H}_{2} \mathrm{O}_{2}$ in methanol for 30 min to quench endogenous peroxide activity, treated in $10 \mathrm{~mm}, \mathrm{pH}$ 6.0, citrate buffer, and heated by microwave for $10 \mathrm{~min}$ for antigen retrieval. The slides were allowed to cool for $20 \mathrm{~min}$ in the same solution at room temperature and then washed in PBS. Normal serum and antibodies were dissolved in PBS containing $1 \%$ bovine serum albumin. Sections were preincubated for 30 min with normal rabbit or goat serum (Dako, Glostrup, Denmark). AntiDKK1 Abs obtained from Santa Cruz Biotechnology (goat polyclonal; raised against an internal region of human DKK1; 1:100) and from AbCam (goat polyclonal; raised against the $\mathrm{C}$ terminus of DKK1; 1:100), anti-p53 Ab (clone DO-7/BP53-12; 1:2000; NeoMarkers, Fremont, CA), and anti-tau (clone AT8; against hyperphosphorylated tau; 1: 2000; Innogenetics, Gent, Belgium) were incubated $30 \mathrm{~min}$ at room temperature and at $4^{\circ} \mathrm{C}$ overnight. Sections were then washed thoroughly with PBS and incubated at room temperature for $1 \mathrm{hr}$ with the appropriate biotinylated secondary antibody diluted in PBS (rabbit anti-goat Ig, 1:300, Vector Laboratories, Burlingame, CA; 1:200, goatanti mouse Ig, 1: 200, Dako, Carpinteria, CA). Single-label immunocytochemistry was performed using an avidin-biotin peroxidase method (Vector Elite) with 3,3'-diaminobenzidine as a chromogen. Sections were counterstained with hematoxylin or Congo red and mounted with Depex (BDH Chemicals, Poole, UK). Sections incubated without the primary antibody or with preimmune sera were essentially blank.

For double labeling, sections (after incubation with primary Abs; DKK1 and P53 or AT8) were incubated for $1 \mathrm{~d}$ at $4^{\circ} \mathrm{C}$ with Alexa Fluor 568 anti-goat IgG and Alexa Fluor 488 antimouse IgG (1:200; Molecular Probes, Eugene, OR). To block auto fluorescence caused by the presence of lipofuscin pigment in the tissue, sections were stained with Sudan Black B (Merck, Darmstadt, Germany) for $10 \mathrm{~min}$. Sections were then analyzed by means of a laser scanning confocal microscope (Bio-Rad; MRC1024) equipped with an argon-ion laser.

To analyze the percentage of DKK1-positive structures, sections labeled with DKK1 and PHF-tau (AT8) were digitized using an Olympus Vanox microscope equipped with a DP-10 digital camera (Olympus Optical, Tokyo, Japan). Images ( $200 \times$ magnification) from representative fields of the lesion in two double-labeled sections of four $\mathrm{AD}$ cases were collected with an Apple Macintosh Power PC 82 computer. The total number of neurons stained with PHF-tau, as well as the structures double-labeled for DKK1 and PHF-tau, was counted. We calculated the percentages of structures immunoreactive for PHF-tau that also contained DKK1 immunoreactivity.

\section{Results}

\section{Apoptotic death and tau hyperphosphorylation in cultured cortical neurons exposed to $\boldsymbol{\beta} \mathrm{AP}$}

We used pure cultures of rat cortical neurons devoid of astrocytes and other contaminating cells. These cultures respond to $\beta \mathrm{AP}$ (fragments 1-42,1-40, or 25-35) with the activation of an unscheduled cell cycle, which leads to an increased p53 expression and apoptotic cell death within $24 \mathrm{hr}$ (Copani et al., 1999, 2002). Treatment with $25 \mu \mathrm{M} \beta \mathrm{AP}$ enhanced the expression of the proapoptotic protein Bax at times that coincide with the increased expression of p53. The antisense-induced knock-down of p53 prevented the induction of Bax and attenuated neuronal death in $\beta$ AP-treated cultures (Fig. 1). Immunoblots with the PHF-1 antibody (which detects the phosphorylated tau epitopes Ser 396 and 404) showed that a $20 \mathrm{hr}$ exposure to $\beta \mathrm{AP}$ induced an increase in tau protein phosphorylation (Fig. 2). To avoid the fast p53-dependent apoptosis of neurons and allow a full development of tau phosphorylation, $\beta$ AP-treated cultures were exposed to a caspase-3 inhibitor. The caspase-3 inhibitor Z-Asp-Glu-ValAsp-CHO (z-DEVD), which prevented $\beta$ AP-induced apoptosis (percentage of neuronal survival: control, $100 \pm 4.3$; $24 \mathrm{hr} \beta \mathrm{AP}$, $42 \pm 3.3 ; 24 \mathrm{hr} \beta \mathrm{AP}$ plus $\mathrm{z}-\mathrm{DEVD}, 20 \mu \mathrm{M}, 78 \pm 5.2^{*} ; 24 \mathrm{hr}$ $\mathrm{z}$-DEVD, $97 \pm 2.5 ; n=3 ;{ }^{\star} p<0.05$ vs $\beta$ AP alone), mediated amplification of tau phosphorylation (Fig. 2). Tau phosphoryla- 
a

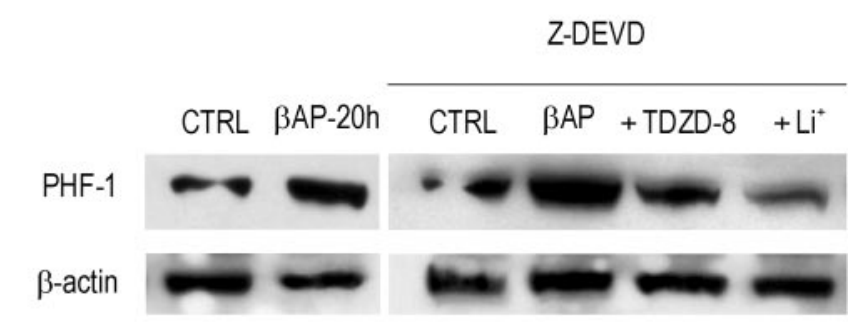

b

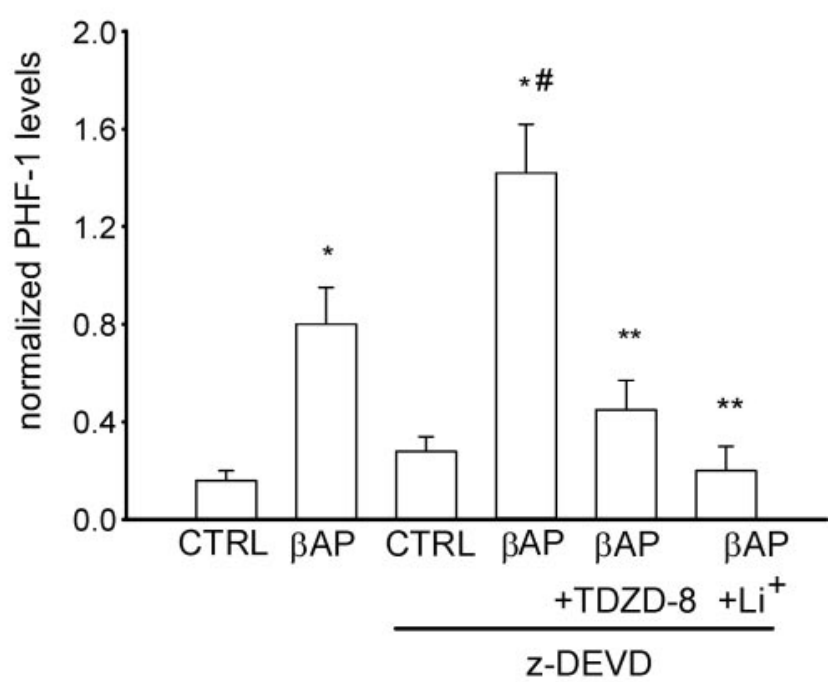

Figure 2. GSK3 $\beta$ inhibitors prevent tau hyperphosphorylation in neurons treated with $\beta A P$ and z-DEVD. $a$, Immunoblot of PHF-1 obtained from neuronal cultures treated for $20 \mathrm{hr}$ with 25 $\mu \mathrm{m} \beta A \mathrm{P}(25-35)$ alone or in the presence of $20 \mu \mathrm{mz}-\mathrm{DEVD} ; 100 \mu \mathrm{m}$ LiCl or $50 \mu \mathrm{m}$ TDZD-8 were added $7 \mathrm{hr}$ after $\beta$ AP. $b$, Densitometer scan analysis from three determinations. Values represent the means \pm SEM of PHF- 1 normalized against $\beta$-actin in three independent experiments. ${ }^{*} p<0.05$ versus controls (CTRL), ${ }^{\#} \beta$ AP alone, or ${ }^{* *} \beta$ AP plus z-DEVD (one-way ANOVA followed by Fisher's LSD test).

tion was prevented by lithium ions added to the cultures $7 \mathrm{hr}$ after $\beta \mathrm{AP}$ (Fig. 2). Although lithium has multiple mechanisms of action, this effect might be attributable to the inhibition of GSK $3 \beta$, an enzyme that phosphorylates tau on the Ser residues recognized by PHF-1 (Godemann et al., 1999). Accordingly, the selective inhibitor of GSK3 $\beta$, 4-Benzyl-2-methyl-1,2,4-thiadiazolidine3,5-dione (TDZD-8) (50 $\mu \mathrm{M})$, reproduced the effects of lithium ions when applied to the cultures $7 \mathrm{hr}$ after $\beta \mathrm{AP}$.

\section{DKK1 is expressed and required for tau phosphorylation in $\boldsymbol{\beta}$ AP-treated neurons}

Searching for a mechanism that could link the increase in p53 expression to GSK3 $\beta$ activation, we examined the expression of DKK1, a protein induced by p53 that negatively modulates the Wnt pathway. Cultures constitutively expressed the DKK1/Wnt coreceptors, LRP5 and LRP6, in addition to several Wnt glycoproteins and the other Wnt coreceptor, Frizzled (Fig. $3 a$ ). In addition, we could detect a functional response to an overexpressed Wnt member (Wnt7A) in cultures that were cotransfected with a reporter gene (luciferase) under the control of a TCF/lymphoid enhancer factor responsive promoter (Fig. $3 b$ ). Thus, the Wnt a

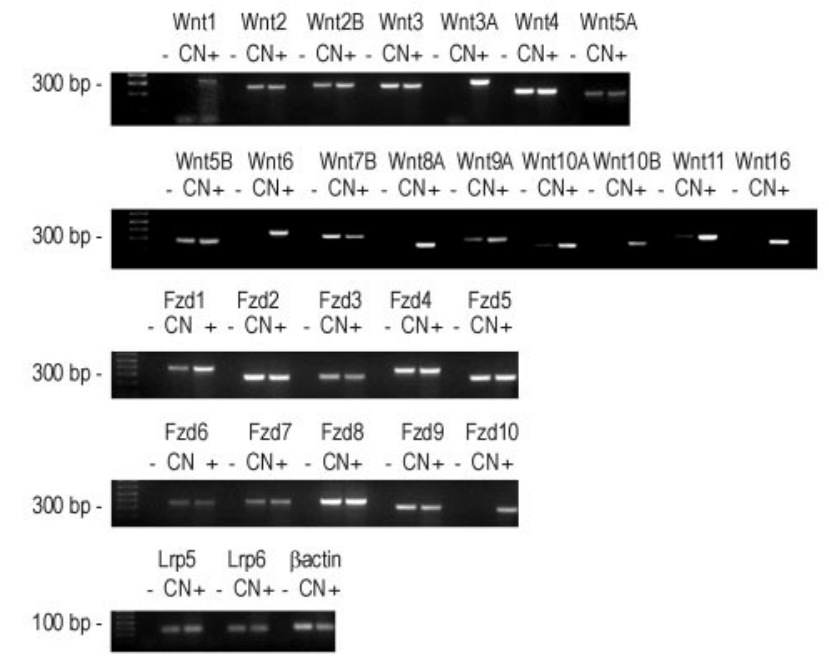

b

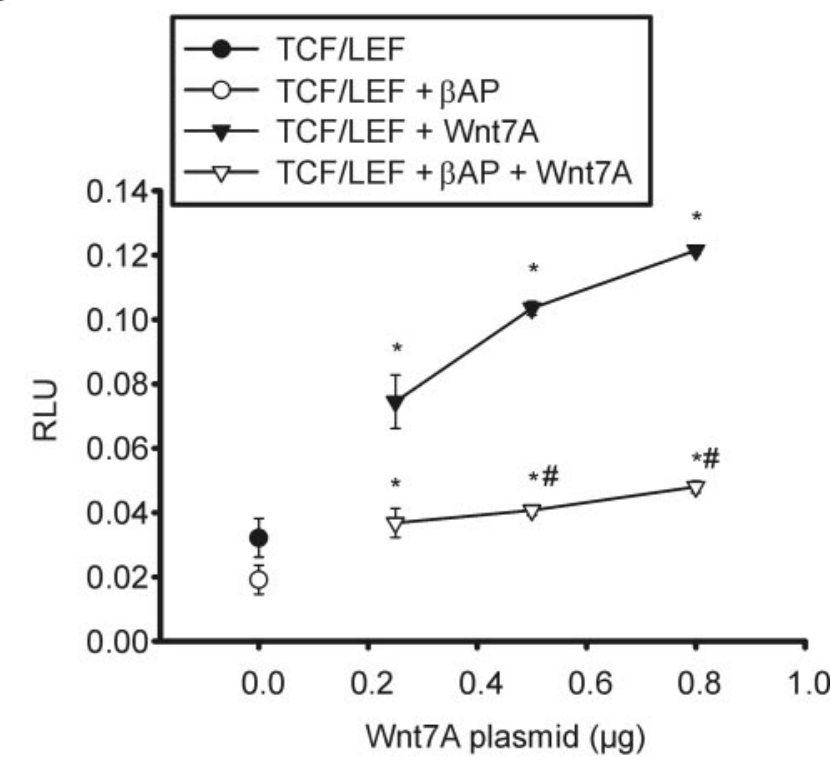

Figure 3. The Wnt transduction machinery is functional in cultured cortical neurons. RT-PCR analysis of mRNA for Wnt glycoproteins, Frizzled receptors, and the DKK1/Wnt coreceptors Lrp5 and Lrp6 in cortical neurons (CN) is shown in $a .+$, Positive control tissues (mixture of CDNA from brain, heart, liver, or kidney); - , negative controls (absence of $\mathrm{CDNA}$ ). The 75 bp $\beta$-actin band is also shown. $b$, TCF/LEF-based luciferase responses to the overexpression of different concentrations of Wnt7a expression plasmid. Values are means \pm SEM of three determinations normalized against the internal standard. ${ }^{*} p<0.05$ versus the respective basal responses; $\# p<0.05$ versus TCF/LEF plus Wnt7A (one-way ANOVA followed by Fisher's LSD test). Filled and open circles indicate basal luciferase responses in the absence or presence of $\beta A P$, respectively. RLU, Relative light units.

transduction machinery was functional in our cultures. Interestingly, the response to Wnt7A was reduced in $\beta \mathrm{AP}$-treated cultures (Fig. 3b), consistent with the reported inhibition of Wnt signaling by $\beta$ AP (De Ferrari et al., 2003). DKK1, which might account for this reduction, was nearly absent in control cultures but became substantially expressed after $16 \mathrm{hr}$ of exposure to $\beta \mathrm{AP}$ (Fig. 4a). Induction of DKK1 was prevented by p53 antisense oligonucleotides (Fig. $4 b$ ). The antisense-induced knock-down of DKK1 had only a small protective effect against neuronal apoptosis (Fig. $5 b$ ) but prevented both the activation of GSK3 $\beta$, 
a

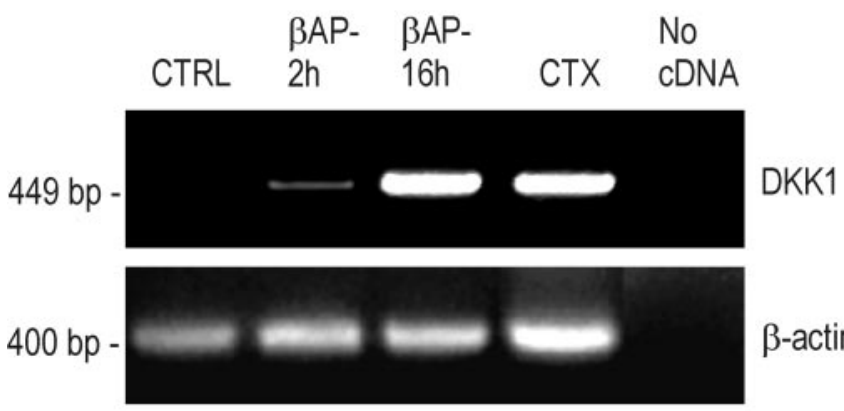

b CTRL $\quad$ AAP $\quad$ p53-Sn $\quad$ p53-As

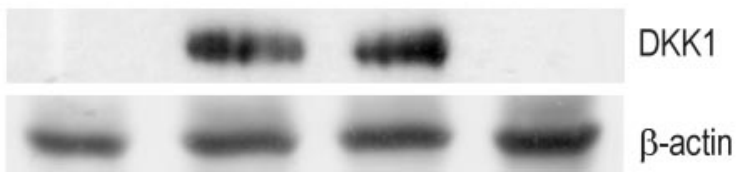

Figure 4. p53-dependent induction of DKK1 by $\beta$ AP in cultured cortical neurons. $a$, RT-PCR analysis of DKK1 mRNA. Cultures were treated with $25 \mu \mathrm{m} \beta \mathrm{AP}(25-35)$ for the indicated times. Expression of mRNA in rat cortex (CTX) is shown as a positive control. The $400 \mathrm{bp} \beta$-actin band is also shown. The $600 \mathrm{bp} \beta$-actin band, assessing genomic DNA contamination, was undetectable. $b$, Representative immunoblot of DKK1 in protein extracts from cortical neurons treated with $25 \mu \mathrm{M} \beta \mathrm{AP}(25-35)$ for $20 \mathrm{hr}$ in the absence or presence of $\mathrm{p} 53$ As or senses (Sn). Loading controls are included.

assessed through the reduction of the inactive phospho-Ser9 form of the enzyme, and the increase in tau phosphorylation that were observed after $21 \mathrm{hr}$ of exposure to $\beta \mathrm{AP}$ (Fig. $5 c$ ). Treatment with DKK1 sense oligonucleotides reduced DKK1 mRNA levels induced by $\beta \mathrm{AP}$ but to a much lesser extent than the respective antisenses (Fig. 5a). However, the extent of DKK1 mRNA reduction produced by DKK1 sense oligonucleotides did not appear sufficient to protect against $\beta$ AP-induced apoptosis (Fig. $5 b$ ) or to prevent both GSK3 $\beta$ activation and tau hyperphosphorylation (Fig. 5c).

\section{Expression of DKK1 in the AD brain}

We extended the examination of DKK1 to human autopsy brain tissue from five $\mathrm{AD}$ patients and five age-matched controls (see Fig. 6 legend). All AD cases displayed a high degree of pathology (Braak score V-VI). Immunohistochemical processing revealed significant DKK1 immunostaining in $\mathrm{AD}$ temporal cortex and white matter (Fig. $6 b, d$ ). Nondemented age-matched controls showed no evidence of DKK1 expression (Fig. 6a,c). Occasional DKK1 staining was observed in glial cells surrounding Congo red-positive plaques (Fig. 6e). In $\mathrm{AD}$ temporal lobe sections that were double immunostained for DKK1 and p53, DKK1 was found in neurons with nuclear p53 expression (Fig. 6f, inset). Neurons with NFT morphology and dystrophic neurites also stained for DKK1 (Fig. 7a,b). In particular, AT8 phosphotaulabeled NFTs, and distrophic neurites were immunoreactive for DKK1 (Fig. 7c,d; Table 1). DKK1-positive white matter fibers also stained for AT8 (Fig. 7e; Table 1).

\section{Discussion}

Recent evidence indicates that abnormalities of Wnt signaling might be involved in human brain diseases, including autism a

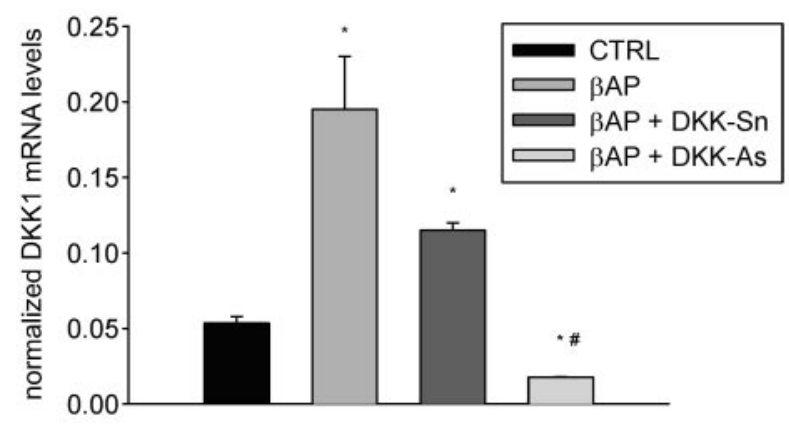

b

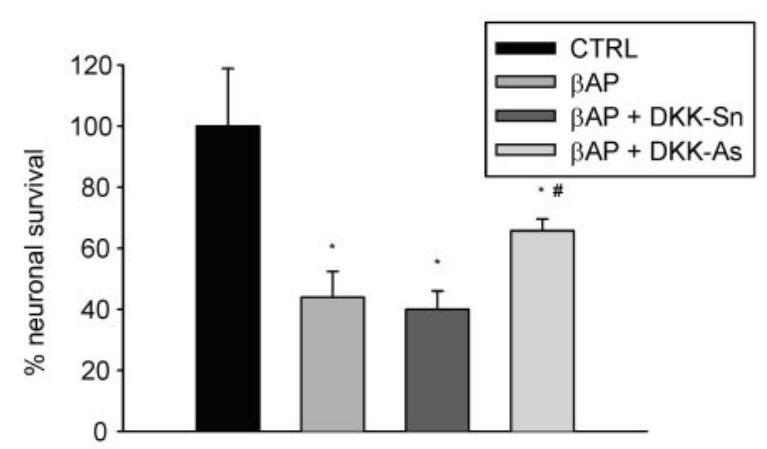

C CTRL $\quad \beta A P+z-D E V D$
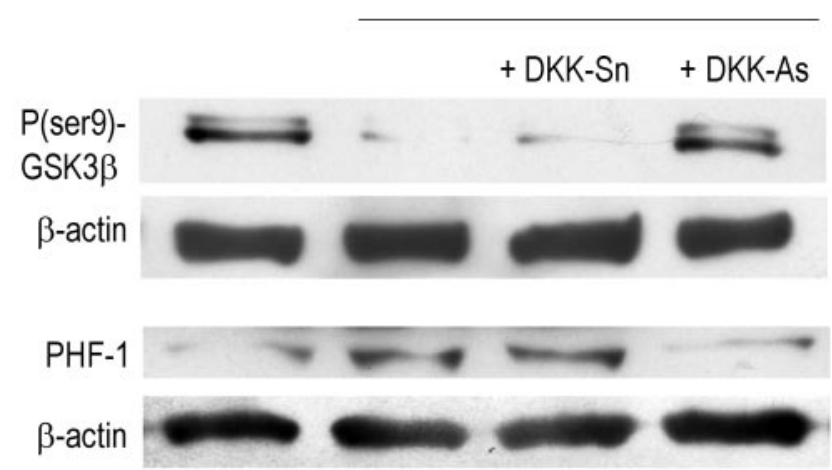

Figure 5. DKK1 knock-down attenuates cell death, GSK3 $\beta$ activation, and tau hyperphosphorylation in $\beta A P$-treated neurons. $a$, Real-time PCR analysis of DKK1 mRNA in neurons exposed to $25 \mu \mathrm{m} \beta \mathrm{AP}(25-35)$ for $16 \mathrm{hr}$ in the presence or absence of DKK1 antisenses (DKK-As) or senses (DKK-Sn). Values are means \pm SEM of normalized DKK1 from four determinations. ${ }^{*} p<0.05$ versus controls (CTRL), or *\# $\beta$ AP alone (one-way ANOVA followed by Fisher's LSD test). The effect of DKK-As on neuronal survival ( $b$ ), GSK3 $\beta$ activation, and tau hyperphosphorylation (c) in cultures exposed to $25 \mu \mathrm{M} \beta \mathrm{AP}(25-35)$ for $20 \mathrm{hr}$ is shown. In $b$, values represent the means \pm SEM of six determinations. ${ }^{*} p<0.05$ versus controls (CTRL), or *\# $\beta$ AP alone (one-way ANOVA followed by Fisher's LSD test). In $c$, the immunoblot of PHF-1 was obtained from neuronal cultures treated with $\beta A P$ in the presence of $20 \mu \mathrm{mz}$-DEVD. The $\beta$-actin band is shown for comparison. The immunoblot is representative of three independent experiments with similar results. The immunoblot of phospho(ser9)-GSK3 $\beta$ was obtained under the same conditions and repeated twice with similar results. 


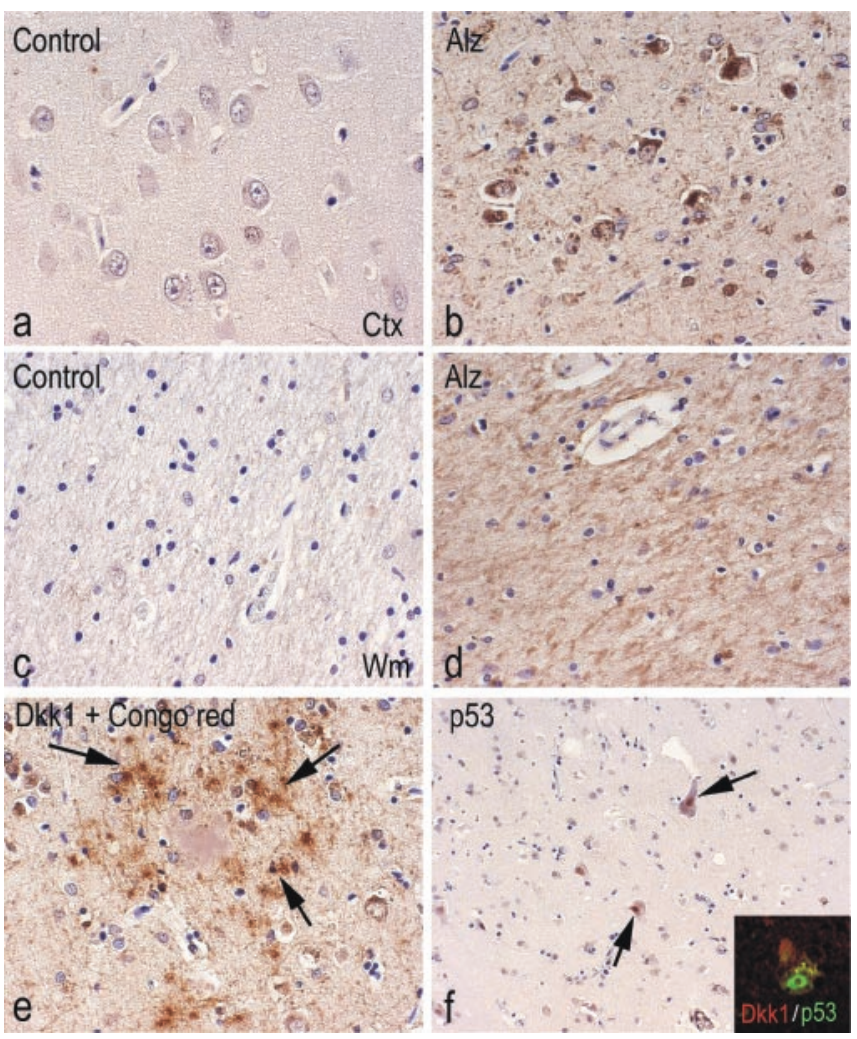

Figure 6. $a-f$, Temporal cortex and white matter immunostained for DKK1 from a representative $A D$ case $(b, d, e, f)$ and an age-matched control $(a, c)$. The examined cases include six nondemented controls (age at death, $77 \pm 4.36$ ), three senile dementia of Alzheimer type (SDAT) (age at death, 85.6 \pm 4.8 ), one familial SDAT (age at death, 76), one AD (age at death, 70 ), and one presenil AD (age at death, 68). DKK1 immunostaining is found in temporal cortex $(\mathrm{Ctx})(b)$ and white matter $(\mathrm{Wm})(d)$ in $\mathrm{AD}$ cases. No staining is observed in nondemented controls $(a, c)$. In e, DKK1-positive glial cells (pointed by arrows) surrounding a Congo redpositive plaque are shown. In $f$, p53-positive cells from temporal cortex of AD are illustrated. $A$ confocal image of DKK1 expression (red) in neurons bearing p53 nuclear expression (green) is shown in the inset. Alz, Alzheimer's dementia.

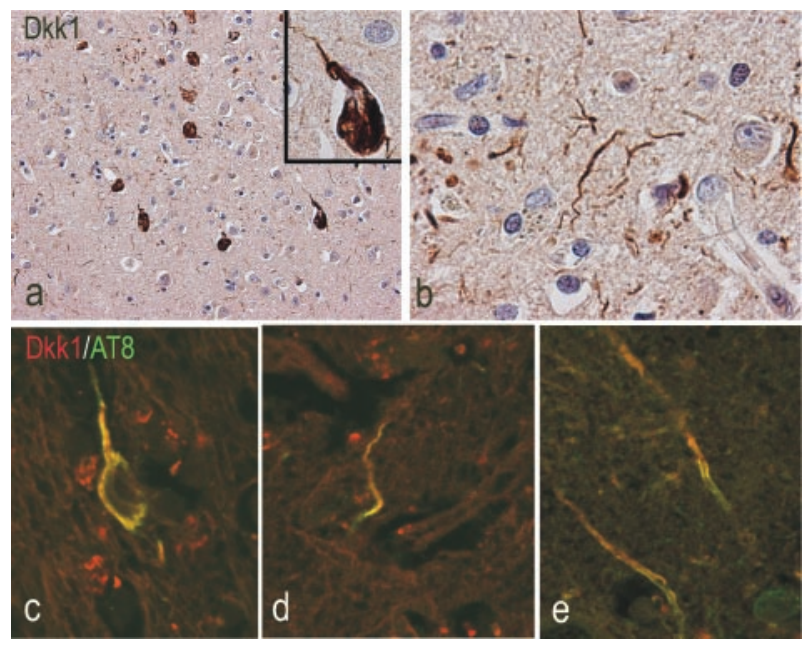

Figure 7. Association of DKK1 expression and hyperphosphorylated tau in AD temporal cortex. $a, b$, Neurons with NFT morphology ( $a$ ) and dystrophic neurites ( $b$ ) stain for DKK1. c, d, The AT8 antibody reveals the location of hyperphosphorylated tau (green) in neurons (c) and dystrophic neurites $(d)$ expressing DKK1 (red). e, AT8 stains also DKK1-positive white matter fibers.
Table 1. DKK1 expression in AT8 phosphotau-labeled NFT and dystrophic neurites

\begin{tabular}{ccc}
\hline & PHF-tau & \\
\hline & Cortex & White matter \\
DKK1 & $85.7 \pm 3.3$ & $79.5 \pm 5.2$ \\
\hline
\end{tabular}

Data represent percentages of structures immunoreactive for PHF-tau that also contained DKK1 immunoreactivity. Data are expressed as means \pm SEM from four AD patients with DKK1-positive structures.

(Wassink et al., 2001), schizophrenia (Cotter et al., 1998), and AD (De Ferrari and Inestrosa, 2000; Garrido et al., 2002; Inestrosa et al., 2002; De Ferrari et al., 2003). In cultured neurons, overexpression of the Wnt antagonist, DKK1, prevents activitydependent dendritic branching (Yu and Malenka, 2003). Our data demonstrate a role for DKK1 in the mechanisms of $\beta A P$ toxicity. In tumor cell lines, DKK1 is induced by p53, which interacts with a potential responsive element located in the promoter region of the DKK1 gene (Wang et al., 2000). Consistent with the notion that DKK1 is a p53 target gene (Wang et al., 2000), induction of DKK1 was also p53 dependent in $\beta A P-$ treated neurons. Thus, distinct $\mathrm{p} 53$-activated pathways of neuronal death occur in response to $\beta \mathrm{AP}$, whereas the expression of Bax might account for the fast execution of apoptosis, the expression of DKK1 might subserve additional roles in the p53activated program. DKK1 knock-down slightly reduced neuronal death induced by $\beta A P$, supporting the hypotheses that the Wnt pathway sustains neuronal survival (De Ferrari and Inestrosa, 2000; Garrido et al., 2002; Inestrosa et al., 2002; De Ferrari et al., 2003), and DKK1 behaves as a proapoptotic factor (Shou et al., 2002). In neurons that were made resistant to apoptosis by inhibition of caspase-3, we could disclose a hyperphosphorylation of tau at times that roughly coincided with the induction of DKK1 (i.e., after $16-21 \mathrm{hr}$ of exposure to $\beta \mathrm{AP}$ ). This particular process was inhibited by DKK1 knock-down and by lithium, which mimics the activation of the Wnt pathway by inhibiting GSK3 $\beta$ (De Ferrari et al., 2003). We speculate that DKK1 produced by $\beta$ APtreated neurons suppresses the canonical Wnt signaling pathway by interacting with LRP5/ 6 and therefore facilitates GSK3 $\beta$ activation. This scenario could take place in degenerating neurons of the AD brain, where DKK1 was highly expressed (whereas it was absent in control brains) and colocalized with both NFT and dystrophic neurites labeled by AT8, which recognizes phosphorylated tau in paired helical filaments. Based on recent findings on the effects of DKK1 overexpression on activity-dependent dendritic branching in neuronal cultures (Yu and Malenka, 2003), DKK1 overexpression in the AD brain would result in deficient neuronal plasticity and, together with its capacity to mediate increased tau phosphorylation (this study), likely contribute significantly to neuronal degeneration. Our demonstration that DKK1 is upregulated in the $\mathrm{AD}$ brain strengthens the hypothesis that an impairment of the Wnt pathway contributes to the pathophysiology of AD (De Ferrari and Inestrosa, 2000; Garrido et al., 2002; Inestrosa et al., 2002; Caricasole et al., 2003; De Ferrari et al., 2003). It is particularly interesting that the DKK1 receptor LRP5 is also one of the putative receptors for apolipoprotein $\mathrm{E}$ (ApoE), and that the genotype $\epsilon 4 / \epsilon 4$ is an established risk factor for late onset AD (Saunders et al., 2000; Rocchi et al., 2003). Whether ApoE4 binds with high affinity to LRP5 and mimics the action of DKK1 on the Wnt pathway is worthy of investigation. Finally, our findings encourage the search for DKK1 antagonist molecules to be tested as selective neuroprotective agents in experimental models of AD. 


\section{References}

Arends M, Duyckaerts C, Rozemuller JM, Eikelenboom P, Hauw J-J (2000) Microglia, amyloid and dementia in Alzheimer disease. A correlative study. Neurobiol Aging 21:39-47.

Bettini E, Magnani E, Terstappen GC (2002) Lithium induces gene expression through lymphoid enhancer-binding factor/T-cell factor responsive element in rat PC12 cells. Neurosci Lett 317:50-52.

Braak H, Braak E (1995) Staging of Alzheimer's disease-related neurofibrillary changes. Neurobiol Aging 16:271-278.

Caricasole A, Ferraro T, Rimland JM, Terstappen GC (2002) Molecular cloning and initial characterization of the MG61/PORC gene, the human homologue of the Drosophila segment polarity gene Porcupine. Gene 288:147-157.

Caricasole A, Copani A, Caruso A, Caraci F, Iacovelli L, Sortino MA, Terstappen GC, Nicoletti F (2003) The Wnt pathway, cell-cycle activation and beta-amyloid: novel therapeutic strategies in Alzheimer's disease? Trends Pharmacol Sci 24:233-238.

Chen R-W, Saunders PA, Wei H, Li Z, Seth P, Chuang DM (1999) Involvement of glyceraldehyde-3-phosphate dehydrogenase (GAPDH) and p53 in neuronal apoptosis: evidence that GAPDH is upregulated by $\mathrm{p} 53$ in neuronal apoptosis: evidence that GAPDH is upregulated by $\mathrm{p} 53$. J Neurosci 19:9654-9662.

Copani A, Condorelli F, Caruso A, Vancheri C, Sala A, Giuffrida Stella AM, Canonico PL, Nicoletti F, Sortino MA (1999) Mitotic signaling by betaamyloid causes neuronal death. FASEB J 13:2225-2234.

Copani A, Sortino MA, Caricasole A, Chiechio S, Chisari M, Battaglia G, Giuffrida-Stella AM, Vancheri C, Nicoletti F (2002) Erratic expression of DNA polymerases by beta-amyloid causes neuronal death. FASEB J 16:2006-2008.

Cotman CW, Anderson AJ (1995) A potential role for apoptosis in neurodegeneration and Alzheimer's disease. Mol Neurobiol 10:19-45.

Cotter D, Kerwin R, al-Sarraji S, Brion JP, Chadwich A, Lovestone S, Anderton B, Everall I (1998) (1998) Abnormalities of Wnt signalling in schizophrenia-evidence for neurodevelopmental abnormality. NeuroReport 9:1379-1383.

Dale TC (1998) Signal transduction by the Wnt family of ligands. Biochem J 329:209-223.

De Ferrari GV, Inestrosa NC (2000) Wnt signaling function in Alzheimer's disease. Brain Res Brain Res Rev 33:1-12.

De Ferrari GV, Chacon MA, Barria MI, Garrido JL, Godoy JA, Olivares G, Reyes AE, Alvarez A, Bronfman M, Inestrosa NC (2003) Activation of Wnt signaling rescues neurodegeneration and behavioral impairments induced by beta-amyloid fibrils. Mol Psychiatry 8:195-208.

Fukumoto S, Hsieh CM, Maemura K, Layne MD, Yet SF, Lee KH, Matsui T, Rosenzweig A, Taylor WG, Rubin JS, Perrella MA, Lee ME (2001) Akt participation in the Wnt signaling pathway through Dishevelled. J Biol Chem 2 76:17479-17483.

Garrido JL, Godoy JA, Alvarez A, Bronfman M, Inestrosa NC (2002) Protein kinase $C$ inhibits amyloid beta peptide neurotoxicity by acting on members of the Wnt pathway. FASEB J 16:1982-1984.

Godemann R, Biernat J, Mandelkow E, Mandelkow EM (1999) Phosphorylation of tau protein by recombinant GSK-3beta: pronounced phosphorylation at select Ser/Thr-Pro motifs but no phosphorylation at Ser262 in the repeat domain. FEBS Lett 454:157-164.
Grimes CA, Jope RS (2001) The multifaceted roles of glycogen synthase kinase 3beta in cellular signaling. Prog Neurobiol 65:391-426.

Hardy J, Selkoe DJ (2002) The amyloid hypothesis of Alzheimer's disease: progress and problems on the road to therapeutics. Science 297:353-356

Hinck L, Nelson WJ, Papkoff J (1994) Wnt-1 modulates cell-cell adhesion in mammalian cells by stabilizing beta-catenin binding to the cell adhesion protein cadherin. J Cell Biol 124:729-741.

Inestrosa N, De Ferrari GV, Garrido JL, Alvarez A, Olivares GH, Barria MI Bronfman M, Chacon MA (2002) Wnt signaling involvement in betaamyloid-dependent neurodegeneration. Neurochem Int 41:341-344.

Lakin ND, Jackson SP (1999) Regulation of p53 in response to DNA damage. Oncogene 18:7644-7655.

Loo DT, Copani A, Pike CJ, Whittemore ER, Walencewicz AJ, Cotman CW (1993) Apoptosis is induced by beta-amyloid in cultured central nervous system neurons. Proc Natl Acad Sci USA 90:7951-7955.

Otth C, Concha II, Arendt T, Stieler J, Schliebs R, Gonzalez-Billault C, Maccioni RB (2002) AbetaPP induces cdk5-dependent tau hyperphosphorylation in transgenic mice Tg2576. J Alzheimer's Dis 4:417-430.

Rocchi A, Pellegrini S, Siciliano G, Murri L (2003) Causative and susceptibility genes for Alzheimer's disease: a review. Brain Res Bull 61:1-24.

Roelen BA, Lin YH, Knezevic V, Freund E, Mummery CL (1994) Expression of TGF-betas and their receptors during implantation and organogenesis of the mouse embryo. Dev Biol 166:716-728.

Saunders AM, Trowers MK, Shimkets RA, Blakemore S, Crowther DJ, Mansfield TA, Wallace DM, Strittmatter WJ, Roses AD (2000) The role of apolipoprotein E in Alzheimer's disease: pharmacogenomic target selection. Biochim Biophys Acta 1502:85-94.

Shen Y, White E (2001) p53-dependent apoptosis pathways. Adv Cancer Res 82:55-84.

Shou J, Ali-Osman F, Multani AS, Pathak S, Fedi P, Srivenugopal KS (2002) Human Dkk-1, a gene encoding a Wnt antagonist, responds to DNA damage and its overexpression sensitizes brain tumor cells to apoptosis following alkylation damage of DNA. Oncogene 21:878-889.

Smale G, Nichols NR, Brady DR, Finch CE, Horton Jr WE (1995) Evidence for apoptotic cell death in Alzheimer's disease. Exp Neurol 133:225-230.

Takashima A, Honda T, Yasutake K, Michel G, Murayama O, Murayama M Ishiguro K, Yamaguchi H (1998) Activation of tau protein kinase I/glycogen synthase kinase-3beta by amyloid beta peptide (25-35) enhances phosphorylation of tau in hippocampal neurons. Neurosci Res 4:317-323.

Wang J, Shou J, Chen X (2000) Dickkopf-1, an inhibitor of the Wnt signaling pathway, is induced by p53. Oncogene 19:1843-1848.

Wassink TH, Piven J, Vieland VJ, Huang J, Swiderski RE, Pietila J, Braun T, Beck G, Folstein SE, Haines JL, Sheffield VC (2001) Evidence supporting WNT2 as an autism susceptibility gene. Am J Med Genet 105:406-413.

Willert K, Nusse R (1998) Beta-catenin: a key mediator of Wnt signaling. Curr Opin Genet Dev 8:95-102.

Yu X, Malenka RC (2003) Beta-catenin is critical for dendritic morphogenesis. Nat Neurosci 6:1179-1187.

Zhang Y, McLaughlin R, Goodyer C, LeBlanc A (2002) Selective cytotoxicity of intracellular amyloid beta peptide1-42 through p53 and Bax in cultured primary human neurons. J Cell Biol 156:519-529.

Zorn AM (2001) Wnt signalling: antagonistic Dickkopfs. Curr Biol 11 R592-R595. 